\title{
Mapping Knowledge Domain Analysis of Physical Activity for Children*
}

\author{
Jinfu Xu \\ Fujian Jiangxia University \\ Fuzhou, China 350108
}

\begin{abstract}
In order to explore the current situation and research evolution of infant sports in China, the year distribution, author distribution, institutional distribution and subject classification of 638 articles related to infant sports in CNKI database from 2000 to 2018 were visualized by Citespace software. The results showed that the physical activity for children is developing rapidly after 2011. Secondly, there is a lack of cooperation between the study authors. Thirdly, the main research institutions are normal colleges and sports colleges, and the network of institutional cooperative relations is forming initially. Lastly, the research subjects mainly focus on the two clustering knowledge groups including infant physical fitness and infant physical education.
\end{abstract}

Keywords-physical activity for children; physical fitness; knowledge maps

\section{INTRODUCTION}

According to the National Statistical Report on Education Development of 2017, the number of children in kindergarten for preschool education in China is 19,379,500 and the number of children in kindergarten is 4,601,000, accounting for $3.5 \%$ of the total population of China. Infant and children are the hope of a country in the future, and their healthy growth is the common aspiration of society and parents. In 2012, the Ministry of Education issued the "Guidelines for the Learning and Development of Children Aged 3-6", which listed children "s sports activities into the health field; in 2016, the State Council issued the "Healthy China 2030 "e Plan Outline" to point out: "Strengthen the guidance of schools and kindergartens and the aged-nursing institutions for nutrition and health". In 2018, on the National Seminar on Physical Education of Early Childhood, it is pointed out that sports should be gradually incorporated into the education system of early childhood to enhance the popularity of sports for children in China. With the publication of a series of policies, children's sports has received unprecedented attention from the society in China in recent years, and the research results of children's sports have also been enriched. In order to better understand the current research status of children's sports and grasp the research progress of children's sports, a few review articles of research on children's sports emerge, but by the author's subjective judgment, the above research results are influenced by more or less deficiencies in the accuracy of data collection, the integrity of the research content, the reliability of the

*Fund Project: Young and Middle-aged Teacher Education Research Project of Fujian Province (JAS180541) conclusions obtained, and the systematic use of the method [1]. In this study, the objective measurement and quantitative method will be used and the Citespace visual analysis software will be applied to explore the knowledge structure and development trend of the research results of children's sports, purposed to provide advantages for the development of China's early childhood sports research.

\section{RESEARCH METHODS}

In the China Academic Journals Network Publishing General Library (CNKI), the advanced search of journal is used, for which the search conditions are: subject $=$ infant sports, search conditions $=$ precise, time span $=2000-2018$, and search time: July 25, 2017. A total of 665 relevant documents were collected, and after 665 related documents are carefully read, the contribution manuscripts, conference papers and the data unrelated to the research are removed, and finally 638 effective documents are retained, and the documents are downloaded in Ref format.

The collected 638 documents are imported into Citespace software, the time span of $2000-2018$ is set in Citespace software, the time slice is 1 , the clustering word sources are title, abstract, author, keyword, supplementary keyword, and the network cutting method is Pathfinder Network (PFNET, Pathfinding Network). In the analysis of the research topic classification, keywords are taken for the node type; in the analysis of authors, the authors are selected for the node type; in the analysis of agencies, the agencies are taken for the node type.

\section{RESEARCH RESULTS}

\section{A. The Distribution of Document Years}

By analysis of the relationship between the number of papers published in the field of children's sports and the distribution year, the development speed and theoretical level of children's sports research can revealed more intuitively [2]. "Fig. 1" shows the years distribution of 626 articles of infant sports research in CNKI published in the period from 2000 to 2018, from which we can seen that the number of literatures on the research of infant sports in China is generally growing. During the period from 2000 to 2010 , the number of study papers on children's sports in China gets less, showing a slow development trend, with an average annual publication volume of only 9.91 papers. After 2011, the volume of documents has 
shown a rapid growth trend with the change of time, and the documents have risen linearly, with an average annual volume of 66.72 articles. In 2012, the Ministry of Education promulgated the "Guidelines for Learning and Development of Children Aged 3-6", which listed the children"s sports activities in the health field that attracted scholars ${ }^{\text {ee }}$ attention to children"s sports. Therefore, it can be seen from "Fig. 1" that after 2012, the number of research papers on children's sports in China has risen sharply, indicating that Chinese scholars are increasingly enriching the research results of children's sports and their research is becoming more and more mature.

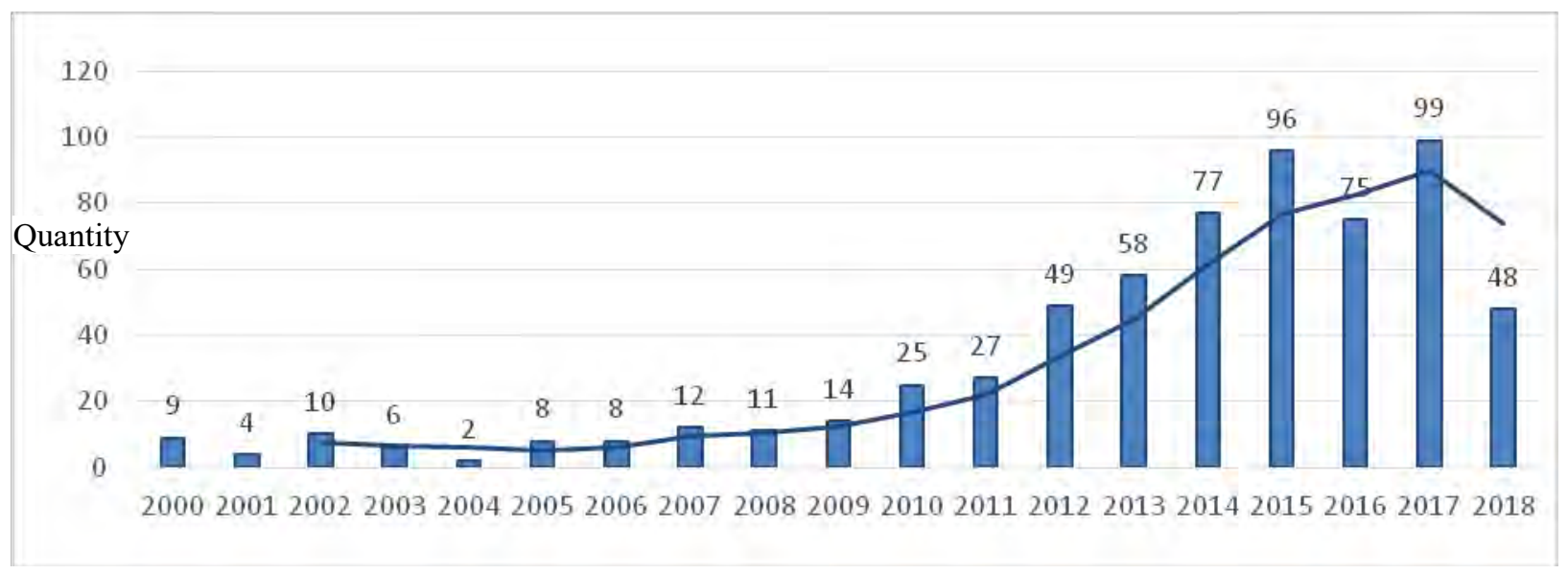

Fig. 1. Year Distribution of research documents on children's sports in China.

\section{B. Distribution of Authors}

The author of the scientific literature is the principal part to promote the progress of the discipline, and it has great significance for the study of the authors of academic papers in a certain field [3]. "Fig. 2" is an author cooperation map for children's sports research that is put out from Citespace. In the figure, each node represents an author, larger the node, more articles the author has published, and the connection lines between the nodes indicate a cooperative relationship among the authors.

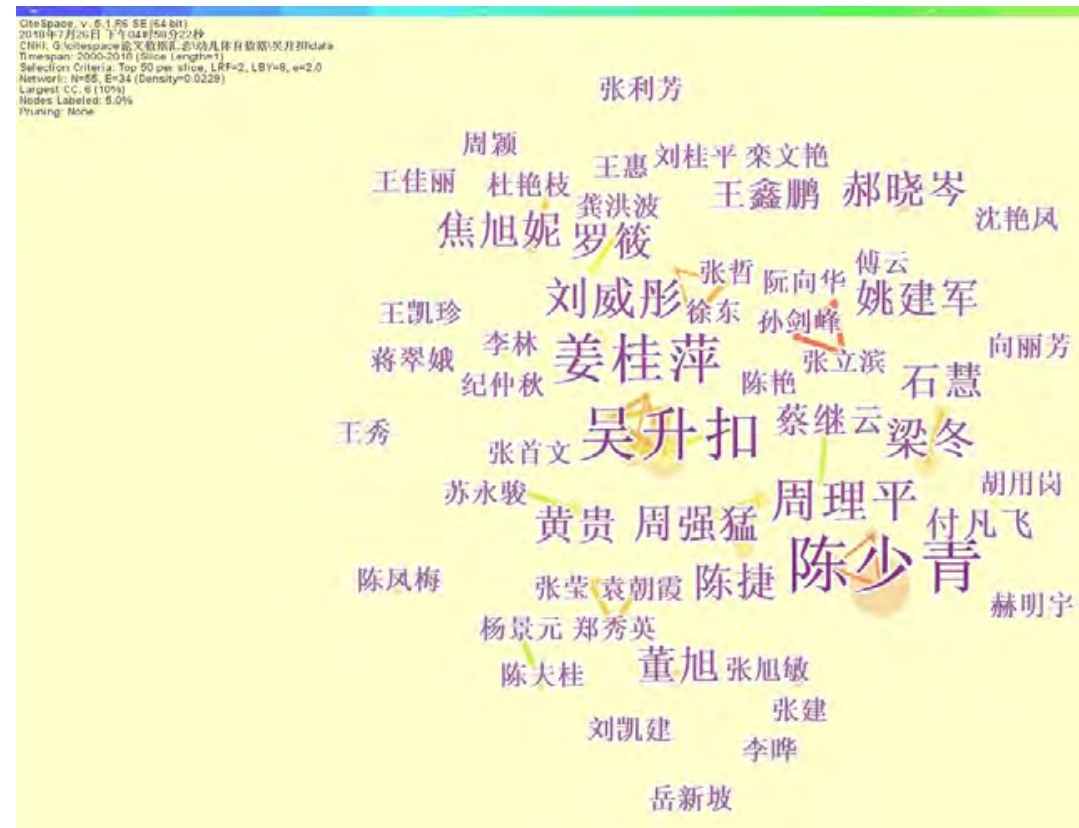

Fig. 2. Author cooperation map for children's sports research.

From "Fig. 2", we can find that Chen Shaoqing has the largest node, indicating that he has published the most papers in the field of children sports research. By statistics of documents, Chen Shaoqing has 12 papers published in 2017, and the research contents focus on the case research of children's sport game group activities; Wu Shengkou has 
published a total of 12 articles from 2014 to the present, mainly conducting empirical research on the physical fitness of children; Hao Xiaocen issued 6 articles. and from the time of writing, we can see Hao Xiaotong is a scholar who earlier conducted early childhood sports research in China, his research content involves the China's children sports right research, the policy analysis and the concept discrimination of children sports, as well as the comparison of children sports development in China and in other countries, and most of his research results are published in Chinese core journals and have been recognized by follow-up scholars; Dong Xu issued 6 articles, with the main research content focused on the teaching practice of children's physical education; Huang Gui issued 4 articles, focusing on the study on various thoughts of scholars on children's sports such as Montessori, Chen Heqin and Frobel; Liang Dong published 3 articles, with the research content mainly focusing on the analysis of the current kindergarten sports activities in Hainan Province and the status quo of preschool sports teachers; Wang Kaizhen issued 3 articles, with the research content mainly focusing on the analysis of children's sports activities. Through the analysis of "Fig. 2" and in combination with reading of relevant literature, the research on children's sports in China has attracted more scholars' attention, but it has not formed a representative author cooperation network, with less cooperation and communication among authors.

\section{Distribution of Research Agencies}

The issuing organization reflects the knowledge generation, creation and dissemination of the research, and through the analysis of the issuing organization, we can evaluate its research strength and its influence in related fields [10]. "Fig. 3" shows a co-occurrence map of children sports research agencies, in which one node represents an agency, and the larger the node, the more the agency's publication volume, and the connection line between nodes indicates a cooperative relationship among the agencies.

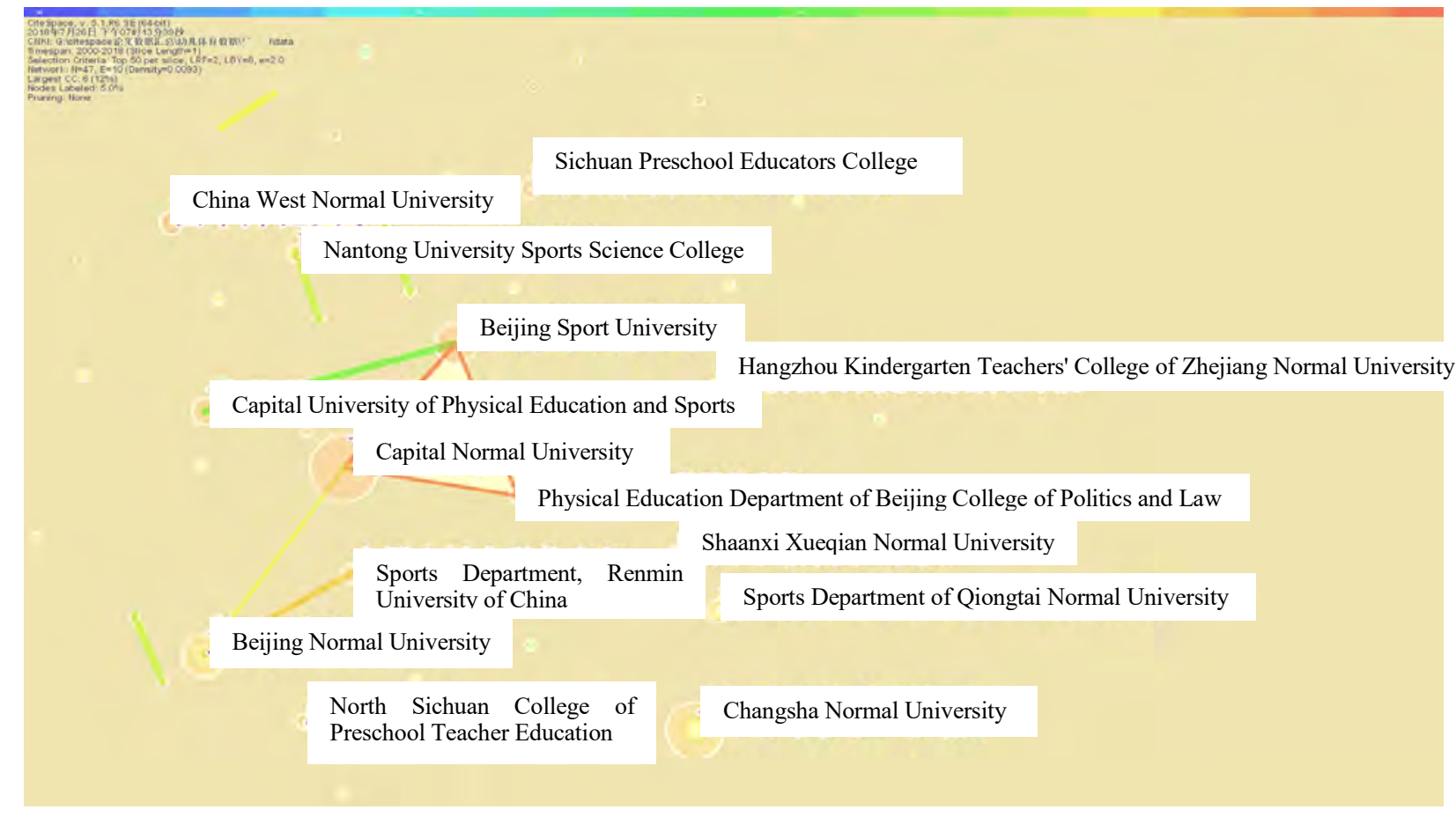

Fig. 3. Distribution of children sports research agencies (frequency $\geq 3$ )

From "Fig. 3", we can see the more obvious research agencies are: Capital Normal University (15 articles), Changsha Normal University (13 articles), Beijing Normal University (10 articles), Beijing Sport University (9 articles), and Capital University of Physical Education and Sports (6 articles) and so on, and there are obvious network connection lines among these agencies, indicating a cooperative relationship in the research in this field. The figure shows that the universities are the main institutions for study of children sports, mainly the teachers' colleges and sports colleges, and the research institutions with cooperative relations are concentrated in Beijing. From the connection lines of the nodes, we can see that the inter-institutional cooperation network for study of children sports has initially shaped in China, and the cross-disciplinary cooperation between the teachers' colleges and the sports colleges has played a role of sharing resource and complementing advantage in the development and promotion of children sports.

\section{Classification of Research Topic}

Among the 7 clusters, the larger the cluster size, the more members in the cluster, and the smaller the cluster number [4]; otherwise, the cluster number is larger. According to the characteristics of 7 clusters, and in combination with the documents, 7 clusters were integrated into 2 cluster knowledge 
groups, of which the first cluster knowledge group is the study of children's physical quality, including \#0 physical energy, \#1 sports quality, \#6 sports deficiency, \#7 physical energy; and the second cluster knowledge group is the study of children's physical education, including \#2 rushing out of Amazon, \#3 sports activity teaching, \#4 countermeasure analysis \#5 children's games.

The knowledge group of the first cluster: the study of children's physical quality. Sports are physical and cultural activities to realize the all-round development of people, with physical activity as the main means; therefore, the study of children's sports must be made in combination with physical fitness. As early as 2000, Huang Wenying et al spent two years in the follow-up experiments on exercise intervention for 3year-old children in kindergarten, and experimental results showed attention to children's daily physical exercise can help improve their physical fitness and develop motor skills.[5] In recent years, with the further and meticulous research, scholars have adopted different interventions to carry out detailed research on the physical quality of children from macro to micro. For example, in 2014, Wu Shengkou elaborated the research progress of the measurement of preschool children's movement development; from 2014 to 2016, made a painstaking study on the development of children's gross movements and physical fitness level from the aspects of static balance ability, rhythmic physical activity, proprioceptive ability and others with 3-6 years old children as the subjects; and in 2017, further studied the integral electromyography contribution rate of the lower limbs of children in side-slide step through the intervention of children movement in the rhythmic physical activities. Numerous studies have shown that as children are in the important stage of growth and development, proper physical exercise of children can not only promote the development of physical quality, but also benefit the development of mental health [6][7].

The knowledge group of the second cluster: the study of children's physical education. The kindergarten education is an important part of school education and lifelong education, and children's physical education is also an important part of the kindergarten education in children's health. According to the literature, among the 638 articles collected, there are 226 articles are related to physical education, accounting for $36 \%$ of the total collected literature, form which, we can see that experts and scholars attach importance to the research of children's physical education. China's physical education in kindergarten has incomplete cognition, arbitrary and single educational contents, absent professional knowledge of teachers, limited venue and equipment in the early stage [8], while now the teaching content is rich and interesting, the teaching form is diversified, and the professional talents are trained and the evaluation criteria of teaching gets reasonable, which all are inseparable from the research of experts and scholars. The research on children's physical education mainly focuses on the current situation of physical education, teacher faculty, sports games and so on, while early research on children's physical education focused on the status quo and countermeasures; scholars analyze the shortcomings and predicaments in children's sports activities, and borrow the advanced foreign development model of sports education to propose the new ideas for the reform of children's physical education in China. With the deepening of research and the good development of children's physical education in China, experts and scholars began to further explore and discuss the diversification and pertinence of children's physical education, and constantly improve the system of children's physical education in China.

\section{CONCLUSION}

In accordance with national policies and documents, China's children sports research has shown a rapid growth trend from the volume of publications in recent years, and the research scholars represented by Chen Shaoqing, $\mathrm{Wu}$ Shengkou, Hao Xiaocen, etc. have carried out detailed research on children's sports from macro to micro and from different angles, but the cooperation is not enough among the authors; research agencies are mainly normal colleges and sports colleges, and the cooperative net is forming initially among the institutions; the research topics are distributed in 7 clusters, and the main two cluster knowledge groups are of children's physical fitness and of children's physical education.

\section{REFERENCES}

[1] WU Xueping, Zhang Jianhua. The Development Characteristics and Hot Spots Change adapting to Sports Research in China[J]. Journal of Shanghai Physical Education Institute, 2014,38(5):5-11. (in Chinese)

[2] Xu Jinfu. A Hot Spot of Research on Physical Activity in China Based on Scientific Knowledge Mapping[J]. Journal of Chongqing Technology and Business University (Natural Science Edition), 2018, 35(3): 123-128. (in Chinese)

[3] Qiu Junping, Yang Siluo, Zhou Chunlei. Analysis of the Authors of the Research Papers on Information Science in China During the 30 Years after Reform and Opening up[J]. Journal of the informatics, 2009, 28(4):626-633. (in Chinese)

[4] Xu Jinfu. Visualization Analysis of Sports Research for Disabled People in China Based on Scientific Knowledge Mapping[J]. Journal of Longyan University, 2017, 35(5): 101-105. (in Chinese)

[5] Huang Wenying, Wang Yan. Analysis of Basic Sports Ability of Children[J]. Jiangxi Social Sciences, 2000, (6): 150-153. (in Chinese)

[6] Wu Shengkou, Jiang Guiping, Li Shugang, et al. An Empirical Study on the Rhythmic Physical Activity in Promoting the Development of Children's Gross Movements from the Perspective of Action Development [J]. Journal of Beijing Sport University, 2015, 38(11): 98105. (in Chinese)

[7] Quan Haiying, Zhang Jingjing, Zhang Ye. Research on the Promotion of Altruistic Behavior of 4-6 Years Old Children by Situational Launching Sports Games[J]. Journal of Shenyang Physical Education Institute, 2014, 33(4): 7-12. (in Chinese)

[8] Wang Yan, Huang Wenying. Study on the Problems and Countermeasures in Kindergarten Physical Education[J]. Jiangxi Social Sciences, 2001,(2):125-126. (in Chinese)

[9] Li Guiqing, Hu Xueqing, Yang Jimei. Analysis of Knowledge Mapping of Aerobics Research in Domestic and Foreign Colleges and Universities [J]. Journal of Wuhan Sports Universiy, 2015,49(2):84-89. (in Chinese) 